\title{
Gestión del aprendizaje para la vida. Una visión holística fundamentada en el aprendizaje autónomo, la neuroeducación y el aprendizaje significativo
}

\section{Managing learning for life. A holistic vision based on autonomous learning, neuroeducation and meaningful learning}

DOI: $10.46932 / \mathrm{sfjdv} 3 \mathrm{n} 1-052$

Received in: Jan 30st, 2021

Accepted in: Feb 1th, 2022

\author{
Liliana Maria Carvajalino Altamar \\ -Psychologist \\ -Doctorate in Educational Sciences. \\ -Master of Education \\ Postgraduate Professor at Universidad de la Costa. Barranquilla, Colombia \\ Cl. 58 \#55 - 66, Barranquilla, Atlántico, Colombia \\ E-mail: lcarvaja4@cuc.edu.co

\section{Dianiris Aurora Rodríguez Colmenares} \\ -Dentist \\ -Doctorate in Educational Sciences. \\ -Specialist in Maxillary Orthopedics \\ -Specialist in Orthodontics \\ Postgraduate Professor at the University of Zulia. Maracaibo Venezuela \\ Science and Health Building, 3rd floor. \\ University of Zulia. Maracaibo Venezuela \\ E-mail: rodriguezdianiris@gmail.com

\section{Edith Lucía González Urdaneta} \\ -Dentist \\ -Dr. in Educational Sciences. \\ -Specialist in Maxillary Orthopedics \\ -Specialist in Orthodontics \\ Postgraduate Professor at the University of Zulia. Maracaibo Venezuela \\ Science and Health Building, 3rd floor. University of Zulia. Maracaibo Venezuela \\ E-mail: edithlucia.31@gmail.com
}

\section{RESUMEN}

En el ser humano los procesos de enseñanza aprendizaje se han desarrollado de distintas formas permitiendo que cada individuo adopte un estilo de aprendizaje particular desconociendo las diversas herramientas con las que puede llegar a construir el conocimiento y a potencializar sus habilidades a lo largo de su vida. Como objetivo fue planteado generar una aproximación teórica para comprender la importancia que tiene la gestión del aprendizaje como una visión holística fundamentada en el aprendizaje autónomo, la neuroeducación y el aprendizaje significativo. La neuroeducación en el ámbito académico puede aportar al desarrollo efectivo de los procesos de aprendizaje para la innovación y transformación de la práctica pedagógica para facilitar el aprendizaje de los educandos en pos de lograr su autonomía, de tal manera que pueda apropiarse de los conocimientos y los pueda llevar a la práctica para entender su entorno y aportar en éste significativamente. Metodología: El presente artículo concierne 
a una revisión descriptiva lo que permitió realizar un estudio detallado, selectivo y crítico con relación a la bibliografía publicada respecto a las temáticas de estudio. Asimismo, se utilizó la técnica correspondiente a los métodos empíricos de investigación, seleccionando publicaciones de articulos y bibliotecas digitales, que sirvieron de base para construir nuevos conocimientos a partir de los descubiertos. Conclusiones: La gestión del aprendizaje permite que los estudiantes construyan a su propio ritmo el conocimiento por lo que es importante desarrollar e implementar un conjunto de estrategias orientadas a la formación de aprendices autónomos capaces de utilizar el pensamiento, la crítica imaginativa, propositiva y creativa para alcanzar su realización como individuos.

Palabras clave: Gestión, Aprendizaje, constructivismo, Aprendizaje autónomo, Neuroeducación, Aprendizaje significativo.

\begin{abstract}
In the human being, teaching and learning processes have been developed in different ways, allowing each individual to adopt a particular learning style, ignoring the different tools with which he/she can build knowledge and potentiate his/her abilities throughout his/her life. The objective was to generate a theoretical approach to understand the importance of learning management as a holistic vision based on autonomous learning, neuroeducation and meaningful learning. Neuroeducation in the academic environment can contribute to the effective development of learning processes for the innovation and transformation of pedagogical practice to facilitate the learning of students in order to achieve their autonomy, so that they can appropriate knowledge and put it into practice to understand their environment and contribute significantly to it. Methodology: This article concerns a descriptive review, which allowed for a detailed, selective and critical study of the bibliography published on the topics under study. Likewise, the technique corresponding to empirical research methods was used, selecting publications of articles and digital libraries, which served as a basis to build new knowledge from those discovered. Conclusions: Learning management allows students to construct knowledge at their own pace, so it is important to develop and implement a set of strategies aimed at the formation of autonomous learners capable of using thinking, imaginative, propositional and creative criticism to achieve their realization as individuals.
\end{abstract}

Keywords: Management, Learning, constructivism, Autonomous learning, Neuroeducation, Significant learning.

\title{
1 INTRODUCCIÓN
}

Desde el ámbito de la educación es necesario reflexionar, debatir y decidir sobre cómo proporcionar a los estudiantes, un aprendizaje que les permita tomar decisiones de manera consciente y lógica, teniendo en cuenta el contexto educativo donde se desarrolla y se sustenta. En la actualidad, todavía se sigue creyendo que si se tiende a reducir el aprendizaje a la adquisición de conocimientos, en su mayor parte verbales, el alumno aprenderá eficazmente. Pero aprender no es sólo cambiar lo que se dice o se sabe, sino también lo que se hace, lo que se quiere o lo que se es, por lo que los cambios producidos por el aprendizaje no sólo afectan al conocimiento de las personas sino también a su comportamiento. (Pozo, Scheuer, Mateos, \& Pérez, 2006; Pine, Messer, \& St. John, 2001; Sewell-Smith, 2004). 
La pedagogía clasifica distintos tipos de aprendizaje, siendo los más importantes: el repetitivo, el receptivo, por descubrimiento y el significativo (Picardo, 2004). Es por ello que a medida que el conocimiento del funcionamiento del cerebro humano vaya siendo más accesible a los educadores, el proceso de aprendizaje se volverá más efectivo tanto para el docente como para el alumno. Campos (2010). De acuerdo con Gamo (2012) "mezclar las ciencias cognitivas y las neurociencias con la educación, permite desarrollar 'estrategias didácticas', así como 'metodologías' más eficaces. Las estrategias de aprendizaje son especialmente importantes ya que constituyen herramientas para que el estudiante en formación se involucre de forma activa, responsable y pueda autodirigir su propio aprendizaje.

El aprendiz estratégico domina un repertorio de estrategias de aprendizaje, las cuales aplica y puede producir nuevas estrategias en concordancia con las situaciones enfrentadas y las metas concretas a alcanzar. Velázquez et al. (2018). En este contexto, Rue (2009) argumenta que es necesario contar con diversos recursos, profesionales y materiales, en los distintos contextos de aprendizaje, para que los alumnos puedan acceder a un mayor compromiso con aquello que se les propone y hacen. Por ello, las actividades que promueven la autonomía del alumno deben someterse a una cuidadosa reflexión en su selección y diseño, basándose en enfoques de la enseñanza que aspiren a promover un aprendizaje del mayor valor formativo.

Si bien son múltiples los factores que puedan incidir para el éxito académico, el docente constituye, después del contexto sociocultural, el segundo factor importante según la Organización de las Naciones Unidas para la Educación, la Ciencia y la Cultura, UNESCO (2010), esto muestra la importancia que el docente mantenga las estrategias para el aprendizaje y continúe incorporando propuestas innovadoras en la enseñanza. Tapia et al. (2019). Para Mora (2013), la neuroeducación es esa nueva visión de la enseñanza basada en el cerebro, que retoma los conocimientos sobre cómo es su funcionamiento, integrado con la psicología, sociología y la medicina, significa evaluar y mejorar la preparación del docente facilitando el proceso de quien aprende.

\section{FUNDAMENTACIÓN TEÓRICA}

El aprendizaje es un proceso y a la vez un resultado, es una práctica individual y una construcción colectiva que se da en un contexto, en una realidad multidimensional. ¿qué conocimiento se adquiere y por qué, dónde, cuándo y cómo se utiliza ? constituyen preguntas esenciales tanto para el desarrollo de los individuos como de las sociedades. UNESCO (2015)

De acuerdo con Gamo (2012) "mezclar las ciencias cognitivas y las neurociencias con la educación, permite desarrollar 'estrategias didácticas', así como 'metodologías' más eficaces. La 
Neuroeducación es un campo de la neurociencia, nuevo, abierto, que posibilita en el ámbito teórico explicaciones novedosas para profundizar en el conocimiento acerca de las condiciones bajo las cuales el aprendizaje puede ser más efectivo. Es un estudio basado en cómo ocurre el aprendizaje en el cerebro soportada por las investigaciones de las disciplinas: neuroanatomía, neurobiología, neurofisiología, neuroquímica y neuropsicología. Cotto (2009).

La neuroeducación según Mora (2013) es una nueva visión de la enseñanza basada en el cerebro, retoma los conocimientos sobre cómo es su funcionamiento, integrado con la psicología, sociología y la medicina. Neuroeducación según este autor significa evaluar y mejorar la preparación del docente y ayudar y facilitar el proceso de quien aprende. Es por ello que a medida que el conocimiento del funcionamiento del cerebro humano vaya siendo más accesible a los educadores, el proceso de aprendizaje se volverá más efectivo tanto para el docente como para el alumno. Campos (2010).

La neurodidáctica, es una rama de la pedagogía basada en las neurociencias que otorga una nueva orientación a la educación. Es la unión de las ciencias cognitivas y las neurociencias con la educación y tiene como objetivo diseñar estrategias didácticas y metodológicas más eficientes, que no solo aseguren un marco teórico y filosófico, sino que promuevan un mayor desarrollo cerebral, (mayor aprendizaje) en términos que los educadores puedan interpretar. Paniagua (2013).

Por otra parte, Velásquez et al. (2006) afirman que las teorías del aprendizaje son la base de la Neuroeducación (La Teoría Neurocientífica o del Cerebro triuno, la teoría del cerebro total o cerebro base del aprendizaje, la teoría del cerebro derecho versus cerebro, la teoría de las inteligencias múltiples planteadas por Gardner. Así como otras teorías que han surgido o apoyado la Neuroeducación, entre ellas se encuentran: la teoría sociocultural (basada en las intersubjetividades y la Zona de Desarrollo Próximo de Vygotsky), la teoría constructivista, el aprendizaje auto-regulado, etc.

La teoría sociocultural del aprendizaje humano de Vygotsky describe el aprendizaje como un proceso social y el origen de la inteligencia humana en la sociedad o cultura. El tema central del marco teórico de Vygotsky es que la interacción social juega un rol fundamental en el desarrollo de la cognición. Según esta teoría, el aprendizaje toma lugar en dos niveles. Primero mediante la interacción con otros, y luego en la integración de ese conocimiento a la estructura mental del individuo.

La teoría del aprendizaje autónomo afirma que los alumnos capaces de autorregularse son aquellos conscientes de su propio conocimiento y comprensión, es decir, que son capaces de establecer qué saben, y qué no saben y deben comprender. Esta teoría propone que el alumno sea, al mismo tiempo, capaz de analizar su propio desempeño, evaluarlo y actuar en consecuencia de su propia evaluación. La autorregulación del aprendizaje juega un papel fundamental en todas las fases del aprendizaje y tiene el potencial de convertir el aprendizaje en algo más significativo para el alumno. 
De esta manera el aprendizaje autónomo le permite a la persona la adquisición de conocimientos y destrezas las mismas que van a ser revisadas continuamente. Por otra parte se debe asumir el aprendizaje autónomo con espíritu crítico, de manera que podamos permitir el debate y diferentes cuestionamientos, hasta encontrar una respuesta. En resumen, si los estudiantes no quieren aprender, no lo van a hacer.

La autonomía en el aprendizaje es la facultad que le permite al aprendiz tomar sus propias decisiones que lo lleven a regular su propio aprendizaje de acuerdo con los objetivos y resultados por alcanzar y al contexto o condiciones específicas de aprendizaje. Por lo tanto el aprendizaje autónomo lleva al aprendiz a vivir la autorregulación permitiéndole satisfacer exitosamente tanto las demandas de sí mismo, como las externas que se le plantean por parte de los instructores y los proyectos de formación, estrategia que fomenta este tipo de aprendizaje. Arriola, (2001). Para que se realice la construcción del conocimiento en la escuela, es necesario considerar la actividad mental constructiva del estudiante, los contenidos de aprendizaje que representan los saberes culturales construidos socialmente y la función del docente, orientada a enlazar el aprendizaje con el contexto. Villarruel (2012).

El aprendizaje autónomo se refiere al grado de intervención del estudiante en el establecimiento de sus objetivos, procedimientos, recursos, evaluación y momentos de aprendizaje, desde el rol activo que deben tener frente a las necesidades actuales de formación, en la cual el estudiante puede y debe aportar sus conocimientos y experiencias previas, a partir de los cuales se pretende revitalizar el aprendizaje y darle significancia. Para Martín (2011), el aprendizaje autónomo es como la capacidad que tiene un individuo para orientar, dominar, reglar y juzgar su manera de educarse, de modo consciente e intencionado, y haciendo uso de estrategias de adiestramiento para conseguir el propósito o intención deseada.

El aprendizaje autónomo es la modalidad de adiestramiento en la que el aprendiz se responsabiliza de la organización de su labor y de la obtención de las diferentes competencias según su característico compás, etc. Implica aceptar el compromiso y el control del suceso individual de adiestramiento, y las decisiones concerniente a planificación, realización y valoración de la práctica de aprendizaje» (Lobato, 2006, p. 191).

El aprendizaje autónomo como condición para continuar aprendiendo durante toda la vida, si bien se plantea dentro de los ideales formativos y dentro de los perfiles de los educandos, no parece haber tenido un espacio propio en los currículos. Pareciera ser que es un aprendizaje que se da por supuesto o por añadidura, a veces se le traslada a escenarios no formales o no escolares, y muchas veces se le reduce a un conjunto de técnicas de estudio. 


\subsection{TEORÍA DEL APRENDIZAJE SIGNIFICATIVO:}

Se entiende que el aprendizaje significativo es el proceso por el cual una nueva información o conocimiento se relaciona con la estructura cognitiva del individuo que aprende. Rodríguez (2004). Uno de los precursores es el psicólogo estadounidense David Ausubel (1976), el cual explica que para lograr un aprendizaje significativo se debe relacionar un nuevo conocimiento o una nueva información con la estructura cognitiva de la persona. Para Matienzo (2020), la esencia de este tipo de aprendizaje está en la relación no arbitraria y sustantiva de ideas compartidas con algún aspecto relevante de la estructura del conocimiento de la persona (conocimiento previo); es decir, con algún concepto, idea o proposición que ya le resulta significativa y adecuada para interactuar con los nuevos datos.

Asimismo, Ausubel (1976), expresa que la esencia del proceso de aprendizaje reside en que las ideas expresadas simbólicamente son relacionadas de modo no arbitrario y sustancial (no literal) con lo que el alumno ya sabe, entendiendo por relación sustancial y no arbitraria que las ideas se relacionan con algún aspecto existente y específicamente relevante de la estructura cognitiva del alumno, por ejemplo, como una imagen, un símbolo ya significativo, un concepto o cuando el niño considera que la mariposa entra en el grupo de aves por que vuela.

Pozo (1989) considera la Teoría del Aprendizaje Significativo como una teoría cognitiva de reestructuración; para él, se trata de una teoría psicológica que se construye desde un enfoque organicista del individuo y que se centra en el aprendizaje generado en un contexto escolar. Desde este planteamiento, la educacion no puede verse solamente como decisiones que surgen en un ambito educativo unicamente, sino tambien, como procesos de interacción con el mundo que debe fundamentarse bajo acciones en las que intervienen los pensamientos, la decisiones y las prácticas educativas.

Según Ausubel (1976), el aprendizaje significativo y aprendizaje mecánico son los dos extremos de un continuo o dimensión en lugar de constituir posiciones dicotómicas, ya que, habitualmente nos movemos entre una y otra. Afirmando, que el aprendizaje mecánico, es un proceso que no supone interacción entre el nuevo contenido y la estructura cognitiva de los que aprenden o que la supone arbitraria y literal; dada la inexistencia de elementos de anclaje claros y relevantes en la misma, el resultado o producto es un aprendizaje repetitivo carente de significado, mientras que, el aprendizaje significativo es el proceso según el cual se relaciona un nuevo conocimiento o una nueva información con la estructura cognitiva de la persona que aprende de forma no arbitraria y sustantiva o no literal.

Asimismo, el aprendizaje comprensivo o significativo produce cambios más duraderos que el mecánico o por repetición de información. Comprender es, en general, una forma de aprendizaje más eficaz y, por tanto, la enseñanza debe buscar esa comprensión. 
Según Ausubel (1973), cuando los conceptos o las proposiciones tienen significado lógico, pueden relacionarse sustancial y no arbitrariamente con elementos de la estructura cognitiva del individuo, siendo su interacción (si se produce) lo que posibilita su transformación en significado psicológico. Éste, es el significado atribuido por la persona a los contenidos aprendidos, de tal modo que el subsumidor que actuó de anclaje se ha modificado y la información que se recibió se ha interpretado.

Torres, Salavarria y Mera (2021) afirman que la enseñanza debe priorizar y facilitar la comprensión de nuevos conocimientos, su clasificación y correlación, la reflexión, el razonamiento lógico, la transferencia de conocimientos hacia nuevos contextos. Por lo que se puede decir, que cada individuo posee una organización cerebral con unas representaciones implícitas que tienen una naturaleza situada que dependen del contexto, aspecto que nos da ciertas limitaciones, ya que se nos hace difícil transferirlas o adoptarlas en nuevas situaciones, que tienen naturaleza con alto contenido emocional. Esto se evidencia en el aula de clase cuando los profesores dan a conocer sus representaciones por medio de comportamientos, haciendo posible que se identifique su rol.

En el aprendizaje no somos siempre activos, pues en ocasiones, hacemos copias y reproducimos la información, sin interpretarla. En cambio otras veces, es posible transformarla, y darle otra valoración. Es decir, todo depende de las concepciones que tengamos del proceso de aprender y de la manera en que interactuamos simbólicamente con nuestro entorno, mediante la socialización y el lenguaje, aspectos fundamentales para Mead (1905) citado por Blanco (1988) el cual plantea que el individuo por su condición social se relaciona con la cultura, a través de códigos, llenos de significado y cargado de valores.

\section{CONSIDERACIONES FINALES}

El aprendizaje es un proceso que el individuo gestiona día día, incluye la predisposición para aprender, subsumidores, estructura cognitiva, modelos mentales, esquema de asimilación, adquisición, retención del conocimiento, planificación, control, regulación y evaluación. Esta gestión no se debe subvalorar, muy al contrario, se constituye fundamental a la hora de enfrentarnos a este mundo tan lleno de información. No tiene sentido el acto de enseñar sin tener en cuenta la predisposición para aprender. Es fundamental analizar lo que ocurre en el entorno del individuo, validar el conocimiento, la potencialidad, la posición evaluativa y crítica, para integrar la resolución de diversas situaciones de acuerdo a su realidad.

La gestión del aprendizaje para la vida pretende promover transformaciones no sólo en la forma como se dicen las cosas sino también cómo se hacen. Busca que el aprendizaje no sea una acumulación de conceptos, sino que se logren cambios cualitativos que propicien los aprendizajes de valores, desarrollo de capacidades y habilidades en la resolución de problemas. La neuroeducación es 
una herramienta de innovación pedagógica y de evolución para los sistemas educativos, sirve en sus diferentes niveles para conocer mejor el funcionamiento del cerebro y relacionarlo con las bases del aprendizaje, permitiendo con esto seleccionar de forma adecuada los planteamientos metodológicos de las clases y consecuentemente la mejora de los procesos de aprendizaje.

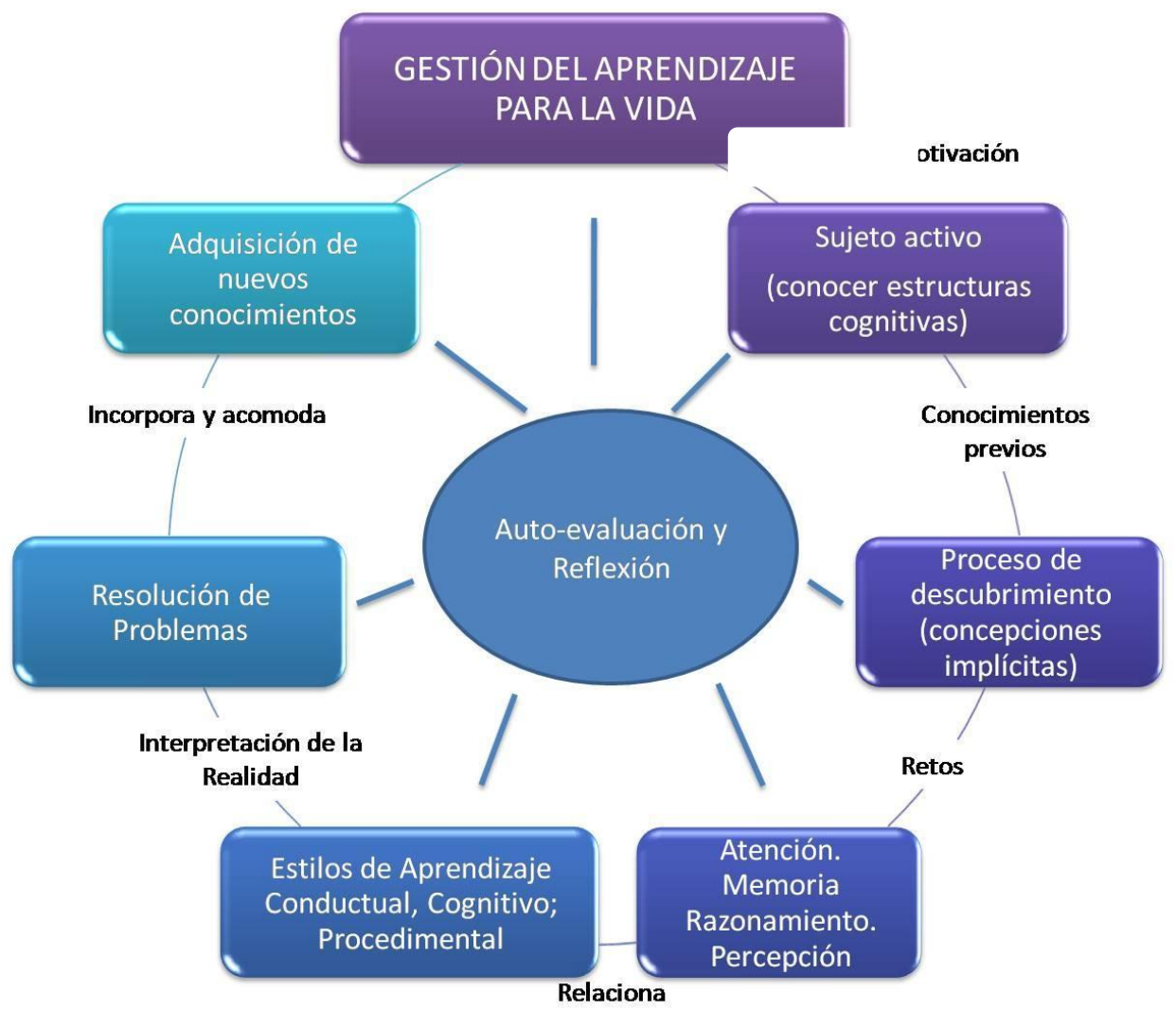

El principio del aprendizaje para la vida es desarrollar la capacidad de aprender cómo aprendo, proceso, registro, almaceno, evoco y respondo a las nuevas experiencias que se presentan en el día a día, es un proceso reflexivo que implica mantener una visión amplia de cambio para poder debilitar las concepciones implícitas previas con las que hemos sido formados y permitir obtener nuevos conocimientos y construir nuevos significados, la neuroeducación abre la puerta, permite abordar diferentes situaciones desde contextos múltiples para la construcción de un sujeto autónomo y autorregulado. 


\section{REFERENCIAS}

Arriola, A. (2001). Relación entre estrategias de aprendizaje y autorregulación. Tesis de grado. México: Universidad Iberoamericana, Ciudad de México.

Ausubel, D. P. (1973). “Algunos aspectos psicológicos de la estructura del conocimiento”. En Elam, S. (Comp.) La educación y la estructura del conocimiento. Investigaciones sobre el proceso de aprendizaje y la naturaleza de las disciplinas que integran el currículum. Ed. El Ateneo. Buenos Aires. Págs. 211-239.

Ausubel, D. P. (1976). Psicología educativa. Un punto de vista cognoscitivo. Ed. Trillas. México.

Blanco, A (1988). Las cinco tradiciones en la psicología social. Capitulo IV: Tradición Institucional. 166223.

Campos A. (2010). Neuroeducación: Uniendo las neurociencias y la educación en la búsqueda del desarrollo humano. (Documento en línea). Disponible en: http://www.educoea.org/portal/La_Educacion_Digital/laeducacion_143/articles/neuroeducacion.pdf

Coll, C. (1992). Introducción: Los contenidos en la Educación Escolar. En C. Coll, J. I. Pozo, B. Sarabia, \& E. Valle, Los Contenidos de la Reforma: Enseñanza y Aprendizaje de Conceptos, Procedimientos y Actitudes. Madrid: Santillana.

Cotto J.(2009). El aprendizaje del cerebro y la educación preescolar. Puerto Rico: Universidad Metropolitana.

Gamo J. (2012). La neuropsicología aplicada a las ciencias de la educación: Una propuesta que tiene como objetivo acercar al diálogo pedagogía/didáctica, el conocimiento de las neurociencias y la incorporación de las tecnologías como herramientas didácticas válidas en el proceso de enseñanza-aprendizaje. (Documento en línea). Disponible en: http://diversidad.murciaeduca.es/publicaciones/dea2012/docs/jrgamo.pdf

Lobato, C. (2006). Estudio y trabajo autónomo del estudiante. En M. De Miguel (Coord.), Metodologías de enseñanza y aprendizaje para el desarrollo de competencias. Orientaciones para el profesorado universitario ante el Espacio Europeo de Educación Superior (pp. 191-223). Madrid: Alianza.

Matienzo, R. (2020). Evolución de la teoría del aprendizaje significativo y su aplicación en la educación superior. Dialektika: Revista De Investigación Filosófica Y Teoría Social, 2(3), 17-26. Recuperado a partir de https://journal.dialektika.org/ojs/index.php/logos/article/view/15

Martín Cuadrado, A. M. (2011): "Competencias del estudiante autorregulado y los estilos de aprendizaje", Revista de estilos de aprendizaje, vol. 4, n.o 8, Madrid, pp. 136-148.

Mora, F. (2013). Neuroeducación. Sólo se puede aprender aquello que se ama. España: Alianza editorial.

Paniagua M. (2013). Neurodidáctica: una nueva forma de hacer educación. Fides et Ratio - Revista de Difusión cultural y científica de la Universidad La Salle en Bolivia, 6(6), 72-77. . (Documento en línea). Disponible

http://www.scielo.org.bo/scielo.php?script=sci_arttext\&pid=S20710081X2013000100009\&lng=es\&tlng $=$ es.

Pozo, J. I. (1989). Teorías cognitivas del aprendizaje. Ed. Morata. Madrid.

Pozo, J. I., Scheuer, N., Mateos, M., \& Pérez, M. P. (2006). Teorías Implícitas sobre el Aprendizaje y la Enseñanza. En J. I. Pozo, N. Scheuer, M. Mateos, M. P. Pérez, E. Martín, \& M. de la Cruz, Las Formas 
de Pensar la Enseñanza y el Aprendizaje: Las Concpeciones de Profesores y Alumnos (págs. 95 - 132). Barcelona: Grao.

Picardo, O. (2004). Diccionario pedagógico, San Salvador: Colegio García Flamenco.

Rodríguez M. (2004). La teoría del aprendizaje significativo. Pamplona- España.

Rué, Joan. (2009). El aprendizaje autónomo en la Educación Superior. Editorial Narcea, 2009, Madrid

Organización de las Naciones Unidas para la Educación, la Ciencia y la Cultura, UNESCO (2010). Factores asociados al logro cognitivo de los estudiantes de América Latina y el Caribe. Laboratorio Latinoamericano de Evaluación de la Calidad de la Educación, LLECE. (Documento en línea). Disponible en: http://unesdoc.unesco.org/images/0018/001867/186769S.pdf

Sewell-Smith, A. (2004). Teaching doesn't necessarily equal Learning. Recuperado el Marzo de 2009, de Teaching Science: The Journal of the Australian Science Teachers Association. blob:https://journal.dialektika.org/d4763532-3117-42d2-baf3-98ac88bd3b75

Tapia C., Márquez L.,. Gaytán L. (2019). Aplicación de las neurociencias en la enseñanza universitaria. caso de una licenciatura en educación. Revista Pedagogía y Sociedad | Vol. 22, no 54, mar. - jun. 2019, ISSN 1608-3784. RNPS: 1903. Estado de Sonora, México.

Torres N., Salavarria B., Mera F. (2021). Estrategias didácticas para mejorar el rendimiento académico en estudiantes de educación superior. South Florida Journal of Development Miami, v.2, n.3, p.39053917special edition, jul. 2021, ISSN DOI:10.46932/sfjdv2n3-008. Miami, USA.

UNESCO (2015) Repensar la educación ¿hacia un bien común universal? www.unesco.org/.../repensar_la_educacion_hacia_un_bien_comun_universal/

Velásquez B., Calle M., Remolina N. (2006). Teorías Neurocientíficas del aprendizaje y su implicación en la construcción de conocimiento de los estudiantes universitarios. Tabula Rasa, 5, 229-245.

Velázquez Y., Nieves O., Rodríguez Y. (2018). Un aprendizaje autónomo de lenguas extranjeras basado en el uso de las estrategias de aprendizaje. Revista Opuntia Brava ISSN: 2222-081x vol. 10 núm. 3. Cuba.

Villarruel M. (2012). El constructivismo y su papel en la innovación educativa. Revista Educación y Desarrollo. 20, 20-28. 